\title{
Master integrals for double real radiation emission in heavy-to-light quark decay
}

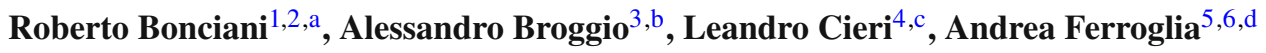 \\ ${ }^{1}$ Dipartimento di Fisica, Sapienza, Università di Roma, Piazzale Aldo Moro 5, 00185 Rome, Italy \\ 2 INFN Sezione di Roma, Piazzale Aldo Moro 2, 00185 Rome, Italy \\ ${ }^{3}$ Physik Department T31, Technische Universität München, James Franck-Straße 1, 85748 Garching, Germany \\ ${ }^{4}$ INFN, Sezione di Milano-Bicocca, Piazza della Scienza 3, 20126 Milan, Italy \\ ${ }_{6}^{5}$ Physics Department, New York City College of Technology, The City University of New York, 300 Jay Street, Brooklyn, NY 11201, USA \\ ${ }^{6}$ The Graduate School and University Center, The City University of New York, 365 Fifth Avenue, New York, NY 10016, USA
}

Received: 9 July 2018 / Accepted: 15 August 2018 / Published online: 23 August 2018

(C) The Author(s) 2018

\begin{abstract}
We evaluate analytically the master integrals for double real radiation emission in the $b \rightarrow u W^{*}$ decay, where $b$ and $u$ are a massive and massless quark, respectively, while $W^{*}$ is an off-shell charged weak boson. Since the $W$ boson can subsequently decay in a lepton anti-neutrino pair, the results of the present paper constitute a further step toward a fully analytic computation of differential distributions for the semileptonic decay of a $b$ quark at NNLO in QCD. The latter partonic process plays a crucial role in the study of inclusive semileptonic charmless decays of $B$ mesons. Our results are expressed in terms of multiple polylogarithms of maximum weight four.
\end{abstract}

\section{Contents}

1 Introduction . . . . . . . . . . . 1

2 Calculation ................ 2

2.1 Kinematics . . . . . . . . . . . . 2

2.2 Auxiliary topologies and master integrals . . . 4

2.3 Differential equations . . . . . . . . . . . . 4

3 Results ................. . . 7

3.1 Alphabet. . . . . . . . . . . . . 7

3.2 Integrals . . . . . . . . . . . . 10

4 Conclusions . . . . . . . . . . . 10

A MIs poles . . . . . . . . . . . . . . . . . 10

References ................. . 11

\footnotetext{
a e-mail: roberto.bonciani@ roma1.infn.it

be-mail: alessandro.broggio@tum.de

c e-mail: cieri@mib.infn.it

de-mail: aferroglia@citytech.cuny.edu
}

\section{Introduction}

The study of inclusive semileptonic $B$ meson decays is important for the determination of the Cabibbo-KobayashiMaskawa (CKM) matrix elements $\left|V_{u b}\right|$ and $\left|V_{c b}\right|$ and, therefore, it constitutes a stringent test on the unitarity of the CKM matrix.

Inclusive determinations of $\left|V_{u b}\right|$ rely on an Operator Product Expansion (OPE) [1-4], according to which the total $B$ meson semileptonic decay rate and various kinematic distributions can be described, at leading order in a power expansion with respect to the inverse $b$-quark mass, in terms of the partonic decay rate of an on-shell $b$ quark into a leptonneutrino pair and a $u$ quark. Within this framework, theoretical predictions for the partonic decay rates are obtained by means of perturbation theory. Phenomenological predictions for observables related to the semileptonic $B$-meson decays are then obtained by combining perturbative calculations for the semileptonic $b$-quark decays with a finite number of nonperturbative parameters.

However, the measurements of the $B \rightarrow X_{u} e \bar{v}$ decay are affected by large backgrounds due to the $B \rightarrow X_{c} e \bar{v}$ decay. In order to suppress this background, experiments impose sharp cuts (for example, cuts on the final state hadronic invariant mass). This in turn leads to problems with the convergence of the OPE in theoretical predictions. These issues can be addressed by parameterizing the residual motion of a $b$-quark in the $B$ meson by means of the shape function [5-7]. Further studies [8,9] showed that with a combination of cuts on the hadronic and leptonic invariant masses the impact of the shape function can be suppressed and the OPE can be used to describe the $B \rightarrow X_{u} e \bar{\nu}$. In both approaches the QCD corrections to the partonic process $b \rightarrow u W^{*}$ (where $W^{*}$ indicates an off shell $W$ boson 
and the mass of the up quark is set to zero) play a crucial role.

An analytic result for the next-to-leading order (NLO) QCD corrections to the $b \rightarrow l \bar{v}_{l} u$ differential decay rate was obtained in [10]. A fully differential calculation of this decay rate at next-to-next-to-leading order (NNLO) was carried out in $[11,12]$. A related result for the differential top-quark semileptonic decay at NNLO was presented in [13]. These NNLO calculations are based on numerical techniques. Analytic results at NNLO are also known; however so far these studies were carried out in the shape-function region by using Soft-Collinear Effective Theory [14-16]. The contribution of these corrections to $\left|V_{u b}\right|$ were then considered in [17].

In this paper, we focus on the analytic calculation of the Master Integrals (MIs) necessary for the determination of the contribution of the QCD double-real radiation to the $b \rightarrow u W^{*}$ decay. This process is one of the three elements that are necessary for the evaluation of the triple-differential distribution in the charged lepton energy, leptonic invariant mass and final-state hadronic invariant mass at NNLO in QCD. For what concerns the other two elements, the twoloop corrections to the $b \rightarrow u W^{*}$ decay were evaluated analytically in [14-16,18], while the one-loop real-virtual contribution will be the subject of future work.

In order to carry out the calculation, we employed the method of reverse unitarity. This approach was introduced in the context of the evaluation of the NNLO QCD corrections to the production of a Higgs boson in gluon fusion [19] and then applied to several other processes (see for example [2025]). The method consists in applying Cutkosky rules [26] in order to map the calculation of the interference between two leading order (LO) $2 \rightarrow 3$ diagrams integrated over the final state phase-space into the evaluation of "cuts" of two-loop $2 \rightarrow 2$ diagrams. In this way, the on-shell condition for the real particles in the final state of the $2 \rightarrow 3$ process is converted into the difference of two propagators with opposite $\mathrm{i}^{+}$prescription. Subsequently, one can calculate the cut diagrams by means of techniques for the analytic evaluation of multi-loop diagrams which were developed starting from the late '90s. In particular, dimensionally regularized scalar integrals are reduced to MIs by using Integration by-Parts Identities (IBPs) [27-29]. Reduction algorithms based on IBPs are implemented in publicly available computer programs [30-38]. The MIs are analytically evaluated by means of the Differential Equations (DE) method [39-43] and expressed in terms of generalized polylogarithms (GPLs) [44-46] of two variables: $t$, which is connected to the invariant mass of the hadronic final state, and $z$, which is related to the leptonic invariant mass. The evaluation of the $\epsilon$ expansion of the MIs was carried out up to terms that include GPLs of maximum weight four.
Our results are also relevant for the determination of the total width of a top quark that decays into a massless bottom quark and a lepton-neutrino pair [13].

The paper is structured as follows. In Sect. 2, we discuss the calculation, by introducing the notation and the kinematics of the process, and by identifying the MIs and the range of validity of their analytic expressions. In Sect. 3, we present the results for the MIs. Section 4 contains our conclusions. "Appendix A" collects the explicit expressions of the $\epsilon$-poles of the MIs.

The analytic expressions of the twelve MIs evaluated in this work are collected in an ancillary file included in the arXiv submission.

\section{Calculation}

The calculation of double emission corrections to the $b \rightarrow$ $u W^{*}$ process is first mapped into the problem of calculating three-particle cuts in two-loop $b W^{*} \rightarrow b W^{*}$ forward box-diagrams, using the method proposed in [19]. Three auxiliary topologies which encompass all of the combinations of denominators which can appear in the cut diagrams were subsequently identified. The MIs belonging to each topology were identified by using IBPs as implemented in LiteRed [31,32]. The MIs, which depend on two dimensionless parameters (defined below), are then calculated by employing the DE method. The technique employed in this work is by now a standard method in the analytic calculation of Feynman diagrams. In this section we describe the way in which we parameterized the kinematics of the process, we define the MIs which were identified and we discuss the way in which the integration constants arising in the DE method were fixed.

\subsection{Kinematics}

At tree-level, the kinematics of the decay we are interested in is

$b\left(p_{1}\right) \rightarrow W^{-}(q)+u\left(p_{2}\right), \quad p_{1}=p_{2}+q$,

where $p_{1}, p_{2}$ and $q$ are the four-momenta of the bottom quark, up quark and $\mathrm{W}$ boson, respectively. Consequently,

$p_{1}^{2}=m_{b}^{2}, \quad p_{2}^{2}=0$

where $m_{b}$ indicates the mass of the bottom quark and the mass of the up quark is neglected. The $W$ boson is off-shell. At tree level the energy of the up quark in the bottom-quark rest frame is smaller that $m_{b} / 2$. Therefore, we introduce the dimensionless parameter $z$ defined as 

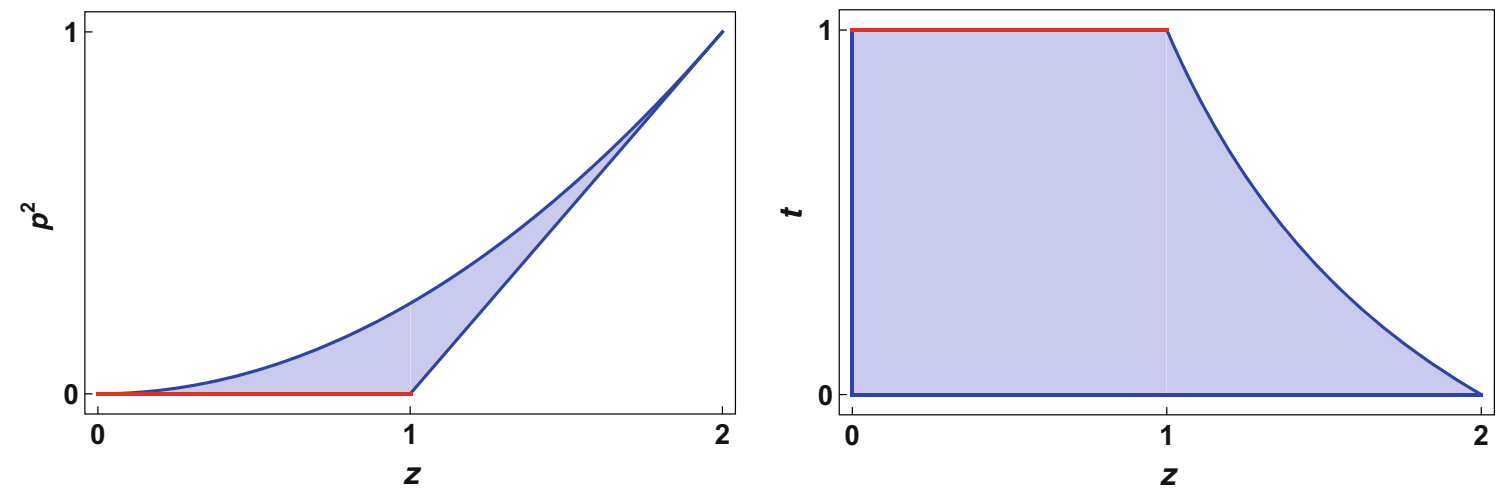

Fig. 1 Physical phase space regions in the $z, \hat{p}^{2}$ plane (left panel) and $z, t$ plane (right panel). In both cases, the line corresponding to tree level kinematics is indicated in red

$z \equiv \frac{2 p_{1} \cdot p_{2}}{m_{b}^{2}}, \quad 0 \leq z \leq 1$

Beyond leading order, it is necessary to consider the process

$b\left(p_{1}\right) \rightarrow W^{-}(q)+X\left(p_{X}\right), \quad p_{1}=p_{X}+q$,

where $X$ indicates an inclusive state involving light quarks and gluons, so that $p_{X}^{2} \neq 0$ in general. As it was done in [10], the invariant mass of the state $X$ is parameterized by introducing the variable $t$ defined through the relation

$p_{X}^{2} \equiv m_{b}^{2} \hat{p}^{2} \equiv \frac{m_{b}^{2}}{4} z^{2}\left(1-t^{2}\right)$

where $z$ remains defined as in Eq. (2.3), provided that $p_{2}$ is replaced by $p_{X}$. Tree-level kinematics, i.e. $p_{X}^{2}=0$ with $0 \leq z \leq 1$, is recovered in the $t \rightarrow 1$ limit. Beyond tree level, the available phase space in the $\left\{\hat{p}^{2}, z\right\}$ and $\{t, z\}$ plane is shown in Fig. 1. The physical region in the $\left\{\hat{p}^{2}, z\right\}$ plane is shown in the left panel of Fig. 1 and it is delimited by the conditions

$0 \leq z \leq 2, \quad \max \{0, z-1\} \leq \hat{p}^{2} \leq \frac{z^{2}}{4}$

In the $\{t, z\}$ plane the physical region (shown in the right panel of Fig. 1) is given by

$0 \leq z \leq 2, \quad 0 \leq t \leq \min \left\{1, \frac{2}{z}-1\right\}$

In the calculation of the MIs which we carry out in this paper, we keep $t \neq 1$. In fact, the differential distributions which we ultimately want to obtain by employing the integrals which we evaluate here is divergent for $t \rightarrow 1$; the divergences are ultimately regulated by "star" distributions of the form ${ }^{1}[10]$

$\left(\frac{\ln ^{n} \hat{p}^{2}}{\hat{p}^{2}}\right)_{*}, \quad n=0,1,2,3$.

However, it is sufficient to calculate the MIs for real radiation corrections by keeping $t \neq 1$, since the expression of the partonic double differential distribution in the $t \rightarrow 1$ limit was evaluated by using Soft Collinear Effective Theory (SCET) in [17]. The partonic real radiation corrections to the complete partonic differential distribution in the $t \rightarrow 1\left(\hat{p}^{2} \rightarrow 0\right)$ limit were recalculated with SCET methods also by us in the preliminary stages of this work and they will play an important role at the moment of assembling the complete differential distributions. In fact these corrections, which receive contributions from double-real and real-virtual diagrams, involve poles in $\epsilon$ and finite terms proportional to $\delta\left(\hat{p}^{2}\right)$ as well as the terms proportional to the star distributions of Eq. (2.8). At the cross section level the coefficients of the star distributions obtained in SCET and the singular terms arising from the calculation carried out at $t \neq 1$ must match; to be specific, in a calculation carried out at $t \neq 1$ one will find divergent terms which should be regulated by star distributions according to relations of the form

$\frac{4}{z^{2}\left(1-t^{2}\right)} \ln ^{n}\left(\frac{z^{2}}{4}\left(1-t^{2}\right)\right) \longrightarrow\left(\frac{\ln ^{n} \hat{p}^{2}}{\hat{p}^{2}}\right)_{*}$.

The calculation of the partonic double differential distribution carried out at $t \neq 1$ depends on the MIs evaluated here

\footnotetext{
$\overline{1}$ Star distributions can be defined through the relation$$
\int_{0}^{\hat{m}^{2}} d \hat{p}^{2} f\left(\hat{p}^{2}\right)\left(\frac{\ln ^{n} \hat{p}^{2}}{\hat{p}^{2}}\right)_{*}=f(0) \frac{\ln ^{n+1} \hat{m}^{2}}{n+1}
$$$$
+\int_{0}^{\hat{m}^{2}} d \hat{p}^{2} \frac{\ln ^{n} \hat{p}^{2}}{\hat{p}^{2}}\left[f\left(\hat{p}^{2}\right)-f(0)\right],
$$

where $f$ is a smooth test function. 
as well as on the two cut MIs which will be the subject of a future work. Therefore, at the stage in which the partonic differential distribution will be assembled, the SCET calculation of the real radiation will serve as a further cross check of the calculation carried out at $t \neq 1$, since the singular terms of the calculation at $t \neq 1$ will have to match the star distributions present in the SCET calculation, after replacements of the type shown in Eq. (2.9). At the same time, the SCET calculation will provide the complete contribution of the real corrections proportional to $\delta\left(\hat{p}^{2}\right)$. We already tested with success this procedure by recalculating at NLO the differential distribution originally derived in [10].

\subsection{Auxiliary topologies and master integrals}

All of the Feynman diagrams contributing to the double emission corrections to the $b \rightarrow u W^{*}$ process can be calculated once the scalar integrals belonging to the (three cuts of the) three auxiliary topologies are known.

The integrals belonging to the first auxiliary topology considered, which is referred to as topology A, are defined as follows

$I_{A}\left(\alpha_{1}, \alpha_{2}, \alpha_{3}, \alpha_{4}, \alpha_{5}, \alpha_{6}, \alpha_{7}\right)=\int \frac{d^{d} k_{1}}{(2 \pi)^{d}} \frac{d^{d} k_{2}}{(2 \pi)^{d}} \prod_{i=1}^{7} \frac{1}{P_{i}^{\alpha_{i}}}$.

The seven propagators $P_{i}$ in Eq. (2.10) are

$$
\begin{aligned}
& P_{1}=k_{1}^{2}, \quad P_{2}=\left(p_{1}-k_{1}\right)^{2}-m_{b}^{2}, \\
& P_{3}=\left(p_{1}-k_{1}-k_{2}\right)^{2}-m_{b}^{2}, \quad P_{4}=\left(p_{2}-k_{1}-k_{2}\right)^{2}, \\
& P_{5}=k_{2}^{2}, \quad P_{6}=\left(k_{1}+k_{2}\right)^{2} \quad P_{7}=\left(p_{2}-k_{1}\right)^{2},
\end{aligned}
$$

where the last three propagators in the list are cut propagators. For example

$$
\frac{1}{P_{5}} \rightarrow \delta\left(k_{2}^{2}\right)=\frac{1}{2 \pi i}\left[\frac{1}{k_{2}^{2}+i 0^{+}}-\frac{1}{k_{2}^{2}-i 0^{+}}\right] \text {. }
$$

Equivalent relations hold for $P_{6}$ and $P_{7}$. As a consequence of the presence of cut propagators, the integrals $I_{A}$ in Eq. (2.10) are zero when at least one among the powers $\alpha_{5}, \alpha_{6}, \alpha_{7}$ is zero or negative. The eight MIs belonging to topology A are shown in Fig. 2. We introduce the following notation in order to label the MIs of topology A:

$$
\begin{aligned}
& I_{1} \equiv I_{A}(0,0,0,0,1,1,1), \\
& I_{2} \equiv I_{A}(0,1,0,0,1,1,1), \\
& I_{3} \equiv I_{A}(0,1,0,0,1,2,1) \text {, } \\
& I_{4} \equiv I_{A}(0,0,1,0,1,1,1) \text {, } \\
& I_{5} \equiv I_{A}(0,1,1,0,1,1,1) \text {, } \\
& I_{6} \equiv I_{A}(0,1,1,0,1,1,2) \text {, } \\
& I_{7} \equiv I_{A}(0,1,1,0,2,1,2) \text {, } \\
& I_{8} \equiv I_{A}(0,1,0,1,1,1,1) \text {. }
\end{aligned}
$$

The integrals belonging to topology B are defined in analogy to Eqs. (2.10, 2.11):

$I_{B}\left(\alpha_{1}, \alpha_{2}, \alpha_{3}, \alpha_{4}, \alpha_{5}, \alpha_{6}, \alpha_{7}\right)=\int \frac{d^{d} k_{1}}{(2 \pi)^{d}} \frac{d^{d} k_{2}}{(2 \pi)^{d}} \prod_{i=1}^{7} \frac{1}{Q_{i}^{\alpha_{i}}}$,

with

$$
\begin{aligned}
& Q_{1}=P_{2}, \quad Q_{2}=\left(p_{1}+k_{2}\right)^{2}-m_{b}^{2}, \\
& Q_{3}=P_{3}, \quad Q_{4}=\left(p_{2}+k_{2}\right)^{2}, \\
& Q_{5}=P_{5}, \quad Q_{6}=P_{6}, \quad Q_{7}=P_{7},
\end{aligned}
$$

where again $Q_{5}, Q_{6}, Q_{7}$ are cut propagators. Topology B involves eleven MIs, which include the eight MIs already needed for topology A, plus the three non planar MIs shown in Fig. 3. The latter are labeled as follows

$$
\begin{aligned}
& I_{9} \equiv I_{B}(0,1,1,0,1,1,1), \quad I_{10} \equiv I_{B}(0,2,1,0,1,1,1), \\
& I_{11} \equiv I_{B}(1,0,1,1,1,1,1)
\end{aligned}
$$

Topology $\mathrm{C}$ is defined by the integrals

$$
I_{C}\left(\alpha_{1}, \alpha_{2}, \alpha_{3}, \alpha_{4}, \alpha_{5}, \alpha_{6}, \alpha_{7}\right)=\int \frac{d^{d} k_{1}}{(2 \pi)^{d}} \frac{d^{d} k_{2}}{(2 \pi)^{d}} \prod_{i=1}^{7} \frac{1}{R_{i}^{\alpha_{i}}},
$$

with

$$
\begin{aligned}
& R_{1}=Q_{2}, \quad R_{2}=P_{3}, \quad R_{3}=Q_{4}, \quad R_{4}=P_{4}, \\
& R_{5}=P_{5}, \quad R_{6}=P_{6}, \quad R_{7}=P_{7} .
\end{aligned}
$$

Topology $\mathrm{C}$ involves five MIs: $I_{1}$ and $I_{4}$, which are present also in topologies $\mathrm{A}$ and $\mathrm{B}, I_{9}$ and $I_{10}$, which are already needed for topology $\mathrm{B}$ and one additional non-planar integral

$I_{12} \equiv I_{C}(1,1,1,1,1,1,1)$

which is shown in Fig. 4.

\subsection{Differential equations}

Each MI satisfies differential equations with respect to the two dimensionless parameters $z$ and $t$ which we use in order to parameterize the phase space. The differential equations can be derived starting from the IBPs and are directly obtained from LiteRed. Only the integrals of topology A involving the propagators $P_{2}, P_{3}, P_{5}, P_{6}, P_{7}$ are found to involve more than two MIs. Indeed, the MIs $I_{5}, I_{6}$ and $I_{7}$ share the propagators listed above and satisfy a system of three coupled first-order differential equations with respect to the variable $t$ and with respect to the variable $z$. (Of course 

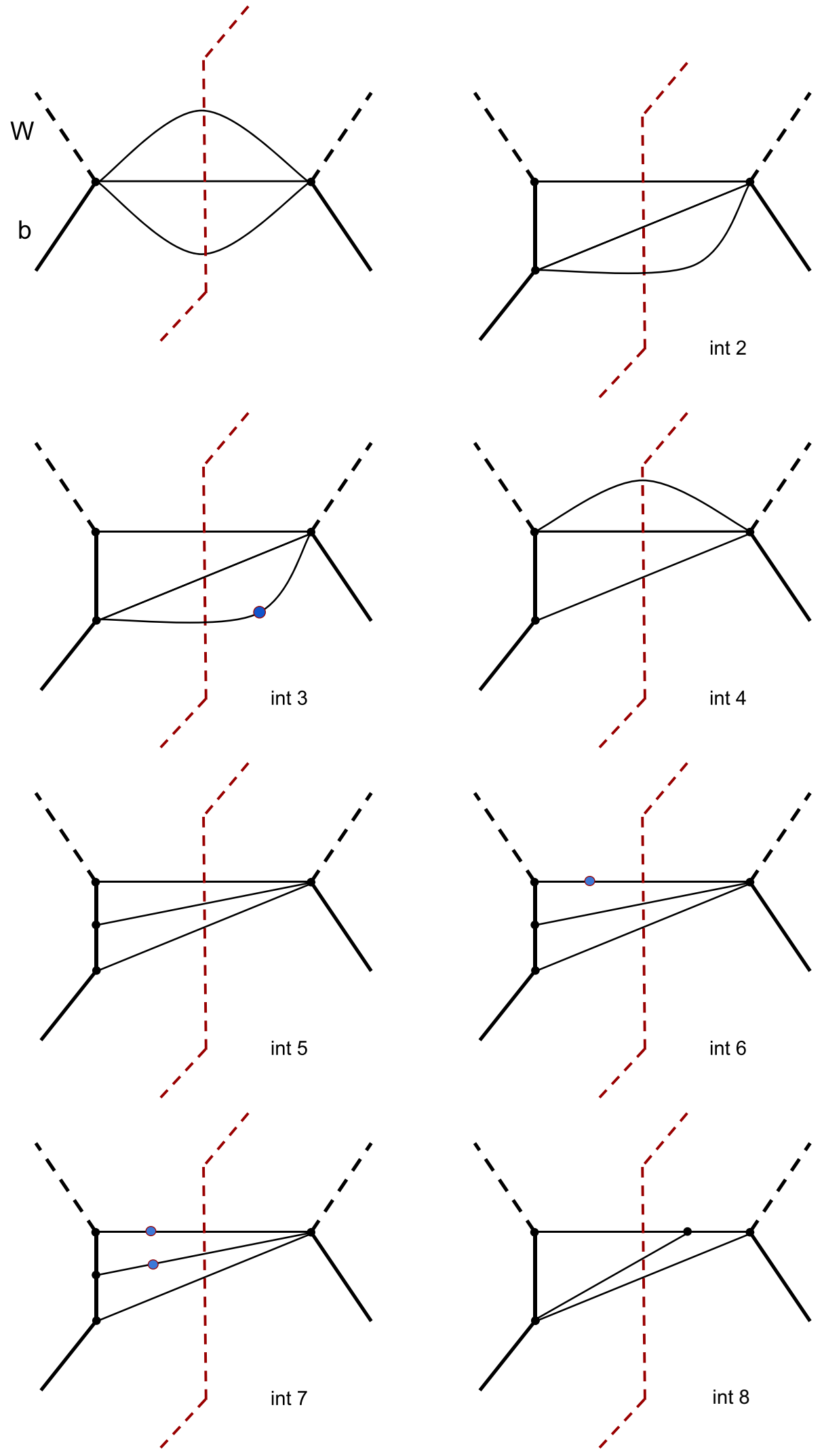

Fig. 2 Master integrals for topology A: integrals $I_{1}, \ldots, I_{8}$ 

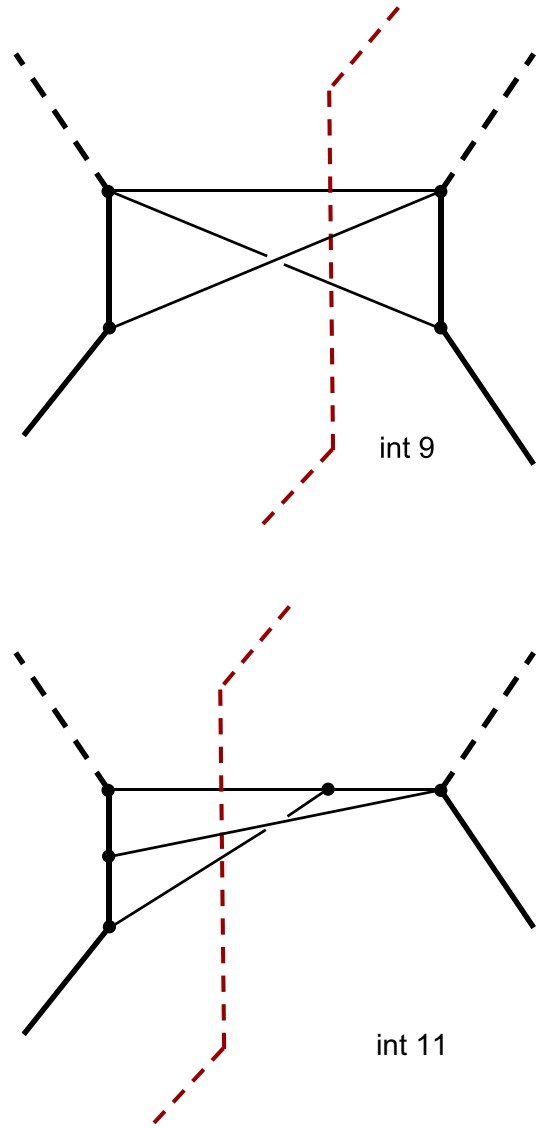

Fig. 3 Additional master integrals from Topology $\mathrm{B}$; integrals $I_{9}, I_{10}, I_{11}$
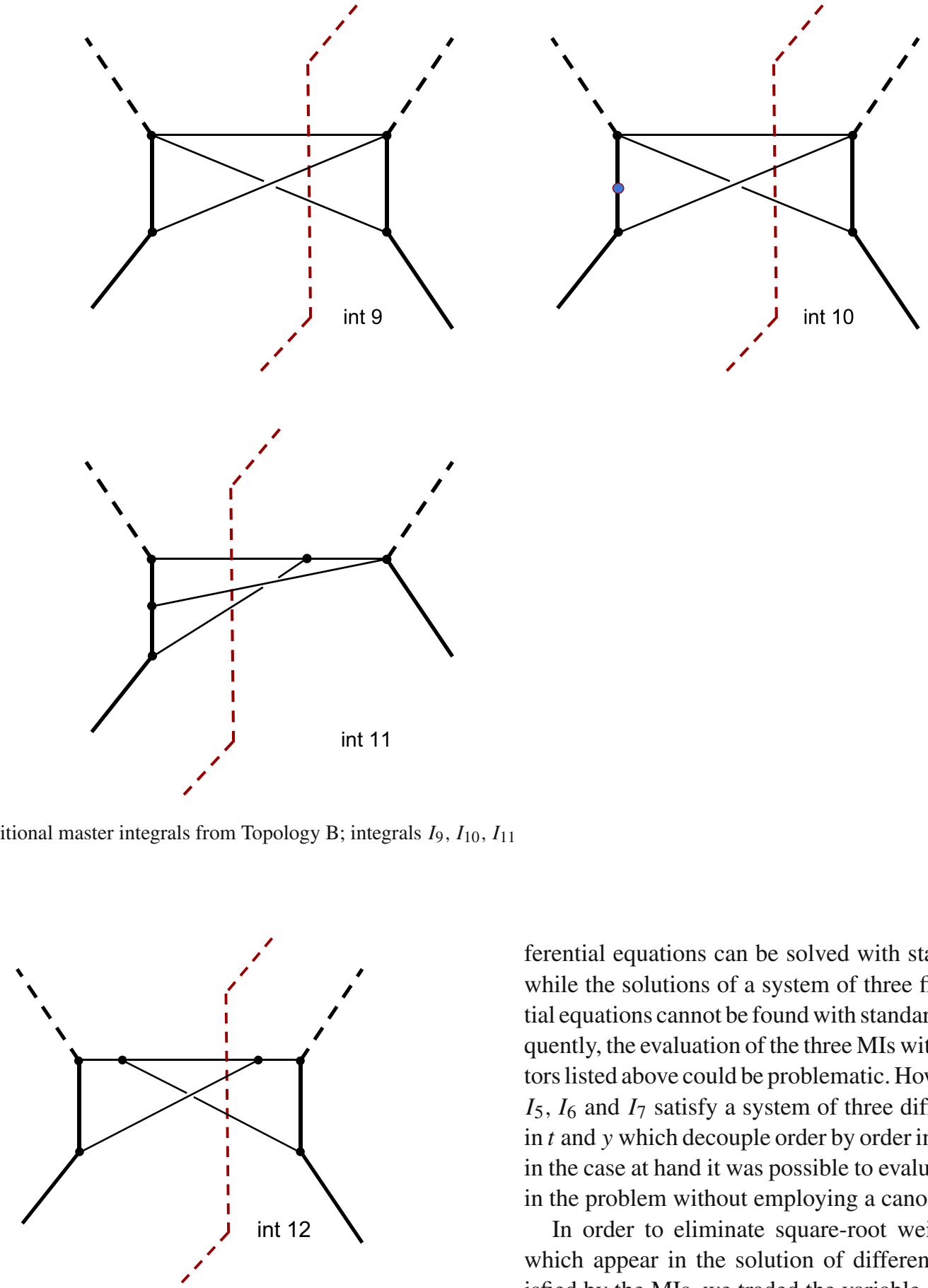

Fig. 4 Additional master integral from Topology C: integral $I_{12}$

the differential equations will also involve MIs depending on a subset of the propagators $P_{2}, P_{3}, P_{5}, P_{6}, P_{7}$. These subtopologies MIs are supposed to be known at the moment of solving the system of equations for $I_{5}, I_{6}$ and $I_{7}$.) The other MIs satisfy either a system of two coupled first-order differential equations (ex. the MIs $I_{2}$ and $I_{3}$ ) or single firstorder differential equations (ex. the MI $I_{8}$ ). First-order differential equations and systems of two coupled first-order dif- ferential equations can be solved with standard techniques, while the solutions of a system of three first-order differential equations cannot be found with standard methods. Consequently, the evaluation of the three MIs with the five propagators listed above could be problematic. However, the integrals $I_{5}, I_{6}$ and $I_{7}$ satisfy a system of three differential equations in $t$ and $y$ which decouple order by order in $\epsilon$. For this reason, in the case at hand it was possible to evaluate the twelve MIs in the problem without employing a canonical basis [47].

In order to eliminate square-root weights in the GPLs which appear in the solution of differential equations satisfied by the MIs, we traded the variable $z$ with the variable $y$ defined through the relation

$z \equiv \frac{(1+y)^{2}}{y}$.

For $0 \leq z \leq 2$ the variable $y$ is a pure phase which we choose to parameterize as

$y \equiv e^{i \alpha}, \quad \frac{\pi}{2} \leq \alpha \leq \pi$.

The differential equations with respect to $t$ and $y$ satisfied by the MIs are solved order by order in $\epsilon$ using iterated 
Table 1 Values of the constants determined by evaluating the analytic result for the MIs with GiNaC. The condition imposed in order to fix the constants is that integrals $I_{2}$ and $I_{5}$ vanish in the $t \rightarrow 1$ limit. The second column indicates the MIs used to fix a given constant and the power $n$ at which the constant first appears as a cofactor of $\epsilon^{n}$. In the last column one can find the complex value of the constant, which can in principle be determined at arbitrary precision

\begin{tabular}{|c|c|c|}
\hline Constant & $\begin{array}{l}\text { Integral/ } \\
\text { order }\end{array}$ & Value \\
\hline$C_{1}$ & $\hat{I}_{2}, \epsilon$ & $\begin{array}{rr} & 10.239578222392149411675195562805 \\
-i \pi & 17.545177444479562475337856971665\end{array}$ \\
\hline$C_{2}$ & $\hat{I}_{2}, \epsilon^{2}$ & $\begin{array}{r}54.371980398832744511559678484441 \\
+i \pi \quad 36.558523291963772395233838229141\end{array}$ \\
\hline$C_{3}$ & $\hat{I}_{2}, \epsilon^{3}$ & $\begin{array}{ll}- & 47.871542821739167668291943163802 \\
-i \pi & 3.3493397758103340833715632589416\end{array}$ \\
\hline$C_{4}$ & $\hat{I}_{5}, \epsilon^{0}$ & $\begin{array}{rr} & 332.70825644476215311648744195295 \\
+i \pi & 177.44567822334599921081142309329\end{array}$ \\
\hline$C_{5}$ & $\hat{I}_{5}, \epsilon$ & $\begin{array}{rr} & 2633.3473713725843348505159328717 \\
-i \pi & 1825.2057515759382789946953393520\end{array}$ \\
\hline$C_{6}$ & $\hat{I}_{5}, \epsilon^{2}$ & $\begin{array}{ll}- & 4672.8756370231810881261045894874 \\
+i \pi & 5234.4198993443246068516773900101\end{array}$ \\
\hline
\end{tabular}

integration. The solutions depend on several integration constants. Most of these constants can be fixed by imposing the regularity of the MIs in the $t \rightarrow 0$ limit. However, a subset of seven constants is left undetermined once the regularity in $t \rightarrow 0$ has been required. MIs are in general not regular in the $t \rightarrow 1$ limit; indeed, one expects the differential distribution to be singular in the tree-level kinematic limit. The singular part of the distribution in the tree-level limit was evaluated by using SCET. However, all of the MIs which are finite in $\epsilon$ (i.e. $\left.I_{1}, I_{2}, I_{4}, I_{5}, I_{9}\right)$ vanish in the $t \rightarrow 1$ limit. In particular, the behavior of $I_{2}$ and $I_{5}$ in the tree level limit is sufficient to overconstrain the seven remaining constants. The analytic expression of the MIs in terms of GPLs of argument $y$ and $t$ are very long but they can be evaluated to arbitrary precision by means of the GiNaC routines of [48]. Therefore, six of the seven constants were fixed by requiring that integrals $I_{2}$ and $I_{5}$ vanish in the $t \rightarrow 1$ limit. They are given in numeric form in Table 1 with more that thirty significant digits. In general, we evaluated MIs up to the order in the $\epsilon$ expansion where GPLs of weight four first appear in the result, since one does not expect GPLs of weight five to be present in the NNLO differential distributions we are ultimately interested in. (Table 2 summarizes the order in $\epsilon$ at which the various MIs were evaluated.) A notable exception is represented by the MI $I_{5}$. Indeed, one of the integration constants which appears alongside GPLs of weight four in $I_{7}$, appears only at order $\epsilon^{2}$ in $I_{5}$. The term of $\epsilon^{2}$ in $I_{5}$ also involves GPLs of weight five. This is not surprising since the set of MIs that was chosen does not have uniform transcendentality. Therefore, we evaluated $I_{5}$ up to order $\epsilon^{2}$ and required that it vanishes in the $t \rightarrow 1$ limit in order to fix this residual constant. In the following section we present analytic results for the MIs.
Table 2 This table summarizes the structure of the $\epsilon$ expansion of the various MIs. The numbers in the table indicate the maximum weight of the GPLs found at a given order of the epsilon expansion. Terms involving GPLs of weight five and higher were not evaluated (except for the case of $I_{5}$, see text) and are indicated with an X in the table

\begin{tabular}{lllllllll}
\hline Integrals & $1 / \epsilon^{2}$ & $1 / \epsilon$ & $\epsilon^{0}$ & $\epsilon$ & $\epsilon^{2}$ & $\epsilon^{3}$ & $\epsilon^{4}$ & Numerical constants \\
\hline$I_{1}$ & - & - & 0 & 1 & 2 & 3 & 4 & None \\
$I_{2}$ & - & - & 1 & 2 & 3 & 4 & $\mathrm{X}$ & $C_{1}, C_{2}, C_{3}$ \\
$I_{3}$ & - & 1 & 2 & 3 & 4 & $\mathrm{X}$ & $\mathrm{X}$ & $C_{1}, C_{2}$ \\
$I_{4}$ & - & - & 1 & 2 & 3 & 4 & $\mathrm{X}$ & None \\
$I_{5}$ & - & - & 3 & 4 & $\mathrm{X}$ & $\mathrm{X}$ & $\mathrm{X}$ & $C_{1}, C_{4}, C_{5}$ \\
$I_{6}$ & - & 1 & 2 & 3 & 4 & $\mathrm{X}$ & $\mathrm{X}$ & $C_{1}, C_{2}, C_{4}, C_{5}$ \\
$I_{7}$ & - & 0 & 1 & 2 & 3 & 4 & $\mathrm{X}$ & $C_{1}, C_{2}, C_{4}, C_{5}, C_{6}$ \\
$I_{8}$ & - & 2 & 3 & 4 & $\mathrm{X}$ & $\mathrm{X}$ & $\mathrm{X}$ & $C_{1}, C_{2}$ \\
$I_{9}$ & - & - & 2 & 3 & 4 & $\mathrm{X}$ & $\mathrm{X}$ & None \\
$I_{10}$ & - & 1 & 2 & 3 & 4 & $\mathrm{X}$ & $\mathrm{X}$ & None \\
$I_{11}$ & 1 & 2 & 3 & 4 & $\mathrm{X}$ & $\mathrm{X}$ & $\mathrm{X}$ & $C_{1}, C_{2}, C_{4}, C_{5}$ \\
$I_{12}$ & 1 & 2 & 3 & 4 & $\mathrm{X}$ & $\mathrm{X}$ & $\mathrm{X}$ & None \\
\hline
\end{tabular}

\section{Results}

\subsection{Alphabet}

The analytic expressions for the MIs which we evaluated are written in terms of Harmonic Polylogarithms [46] and (twodimensional) GPLs [45,48-50] of arguments $t$ and $y$. GPLs can be defined recursively, for $n \geq 0$, via the iterated integral

$G\left(a_{1}, \ldots, a_{n} ; z\right)=\int_{0}^{z} \frac{d t}{t-a_{1}} G\left(a_{2}, \ldots, a_{n} ; t\right)$,

for a generic argument $z$ and weights $\left\{a_{1}, \ldots, a_{n}\right\}$, assuming $G(z)=G(; z)=1$. The case in which $a_{i}=0$ for all $i$ needs to be considered separately:

$G(0, \ldots, 0 ; z) \equiv \frac{1}{n !} \ln ^{n} z$

The weights of the GPLs or argument $t$ can depend on $y$. For convenience we define the combinations

$w_{1} \equiv \frac{1-y}{1+y}, \quad w_{2} \equiv \frac{1+y^{2}}{(1+y)^{2}}=\frac{z-2}{z}$.

Consequently, $w_{2}$ is real while $w_{1}$ is imaginary.

The list of weights, i.e. the "alphabet", appearing in the GPLs of argument $t$ is

$\left\{0, \pm 1, \pm w_{1}, \pm w_{2}\right\}$.

The alphabet for the GPLs of argument $y$ includes the weights

$\{0, \pm 1, \pm i\}$ 


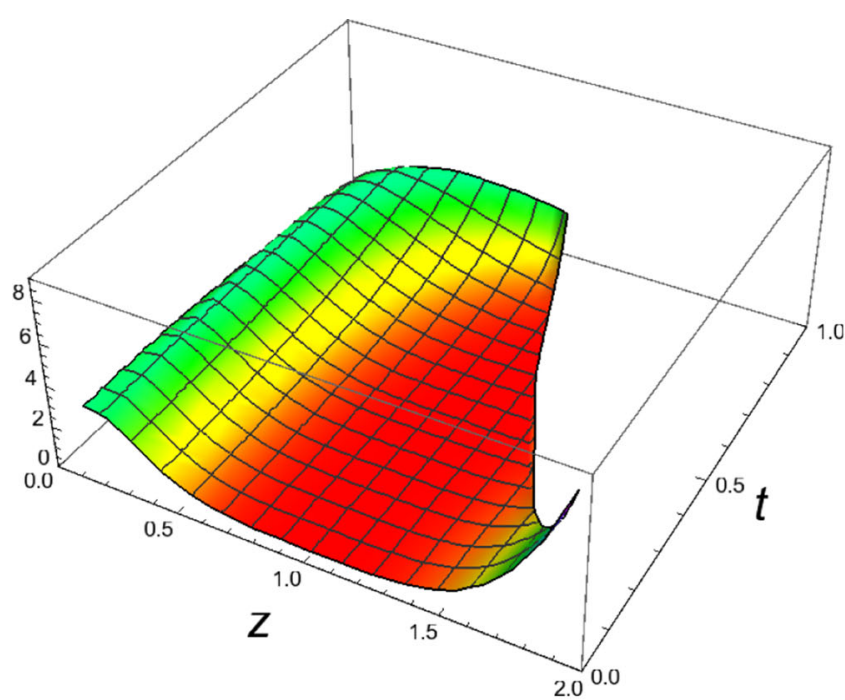

Phase Space integral order $\epsilon^{4}$

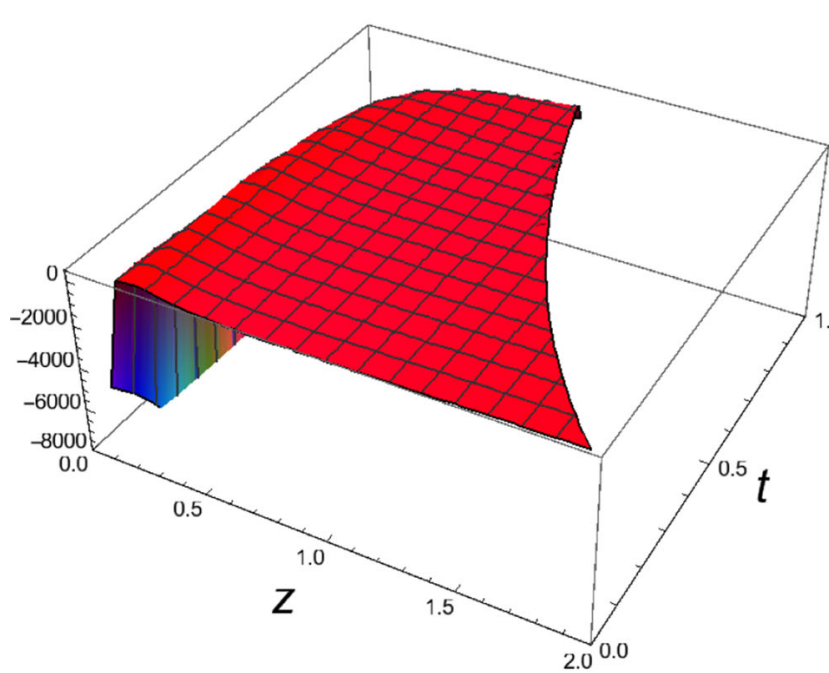

int 3 order $\epsilon^{2}$

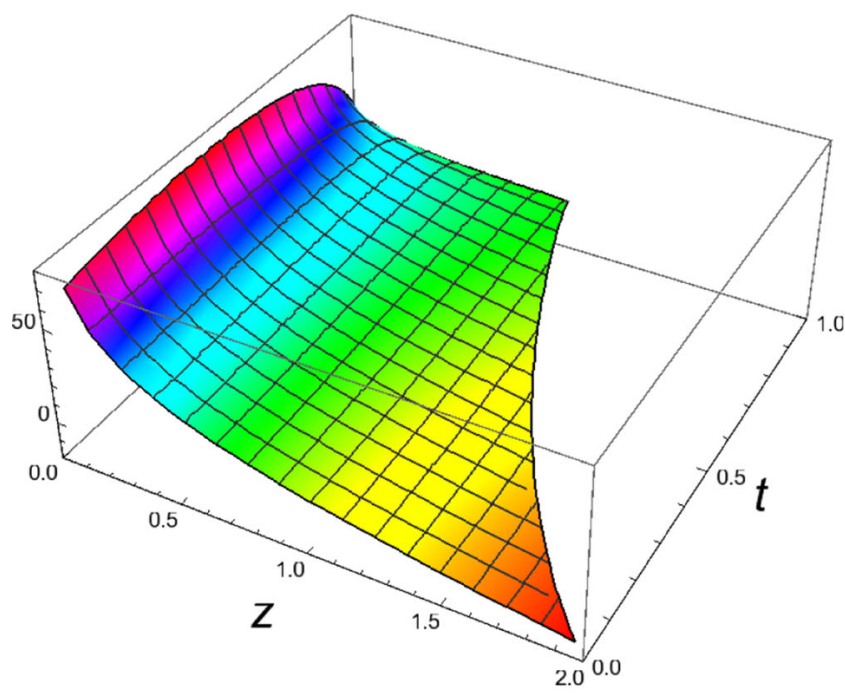

int 5 order $\epsilon$

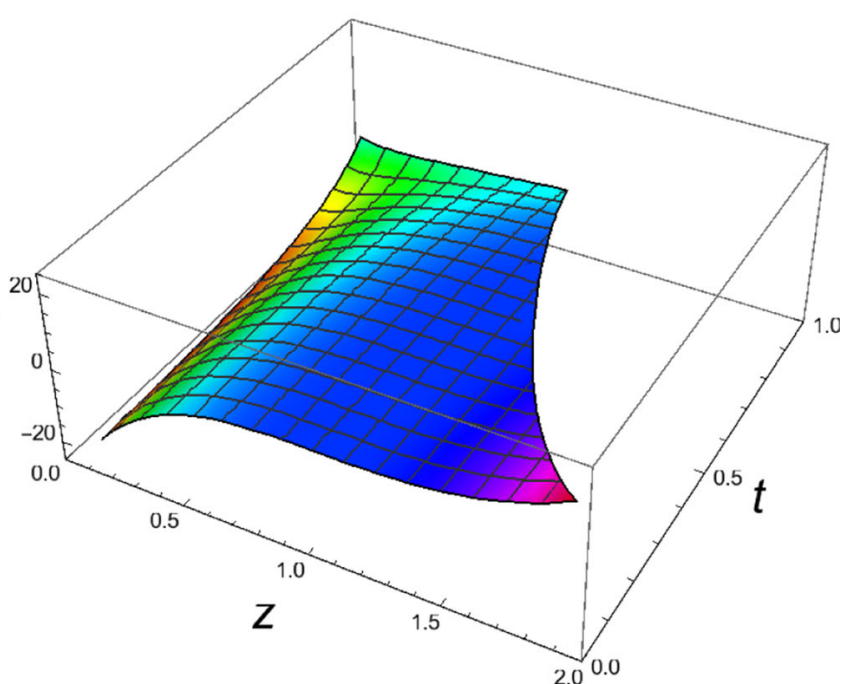

int 2 order $\epsilon^{3}$

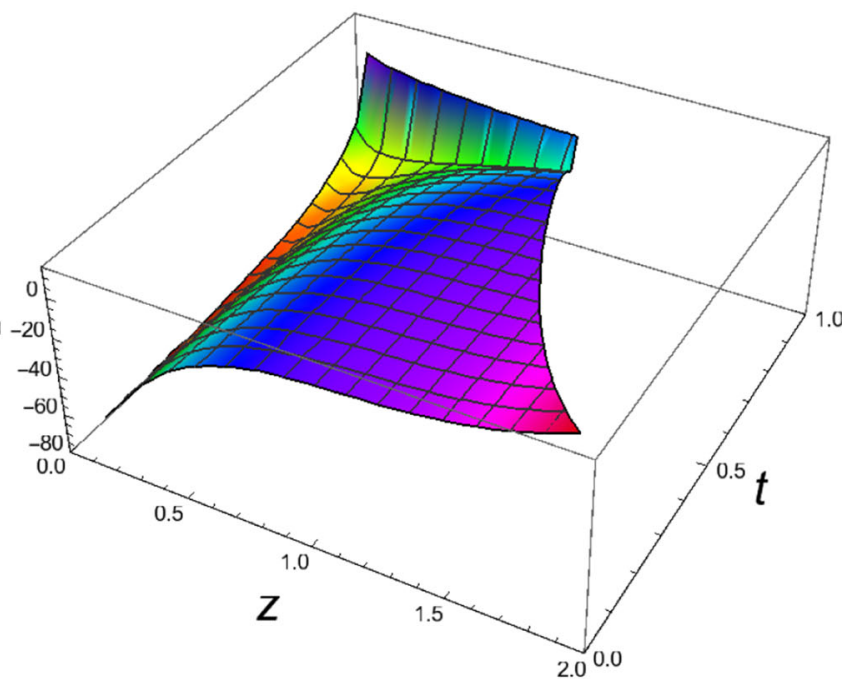

int 4 order $\epsilon^{3}$

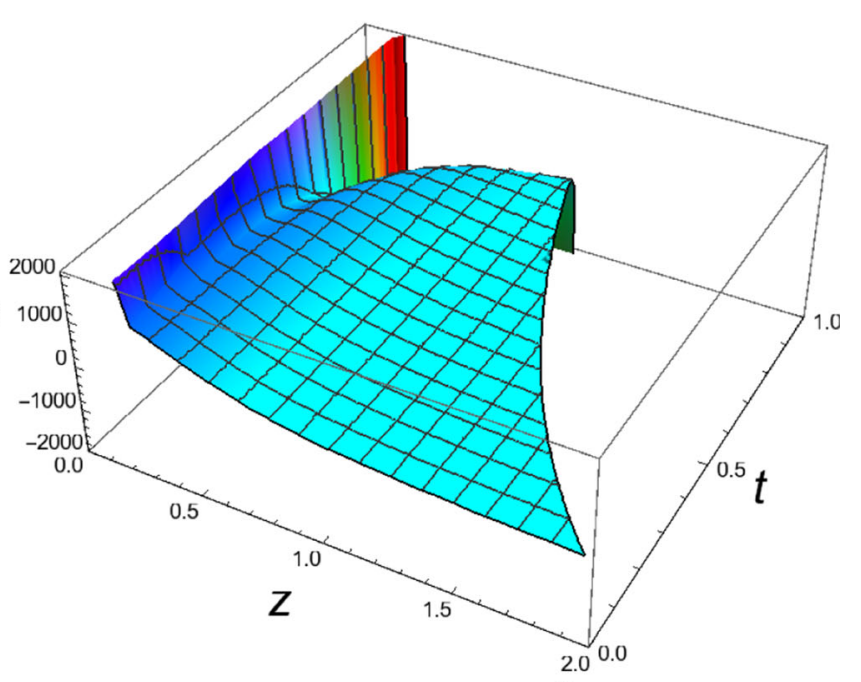

int 6 order $\epsilon^{2}$

Fig. 5 Plots of the first order in $\epsilon$ which contains GPLs of weight four for the MIs $\hat{I}_{1}, \ldots, \hat{I}_{6}$ 


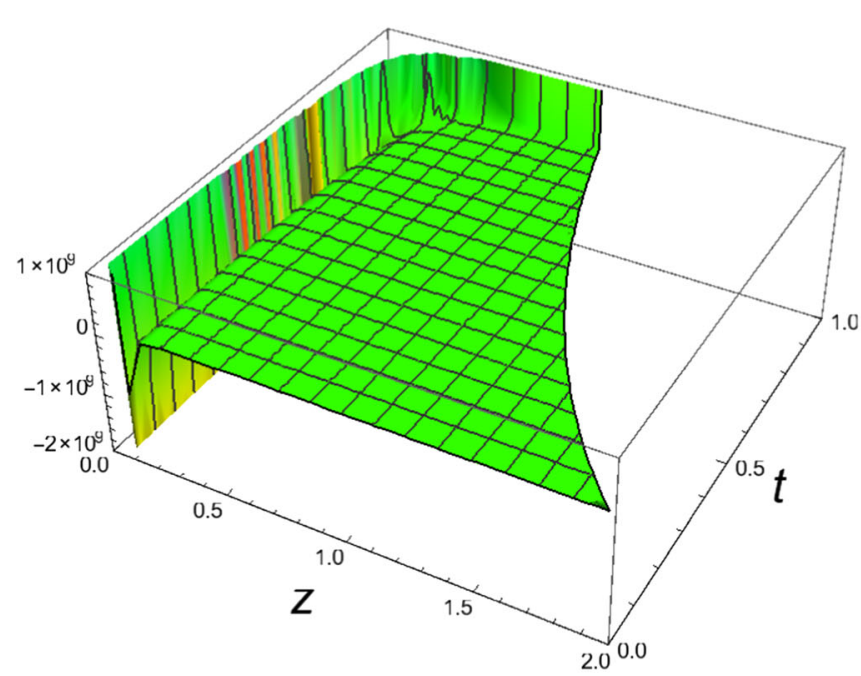

int 7 order $\epsilon^{3}$

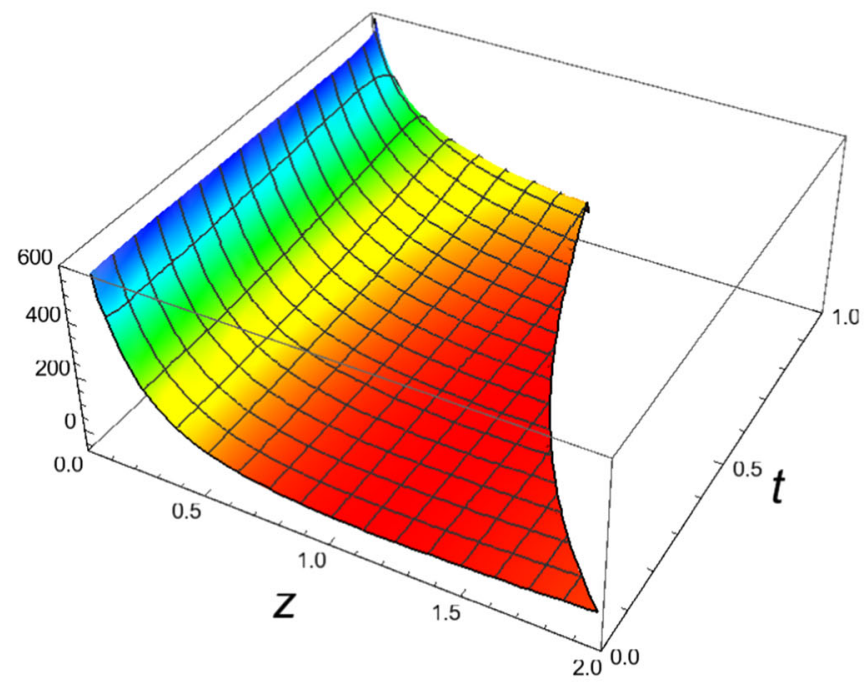

int 9 order $\epsilon^{2}$

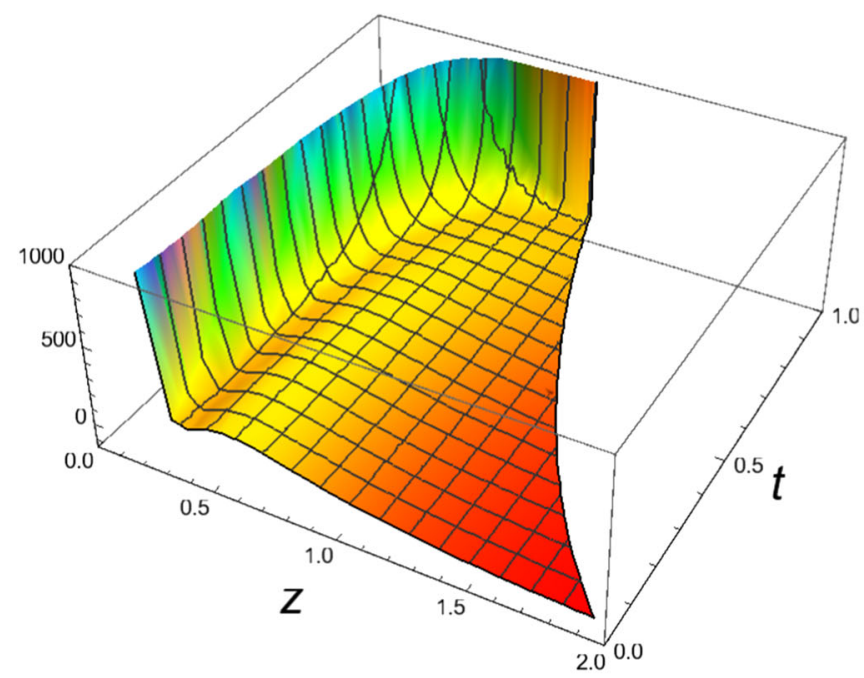

int 11 order $\epsilon$

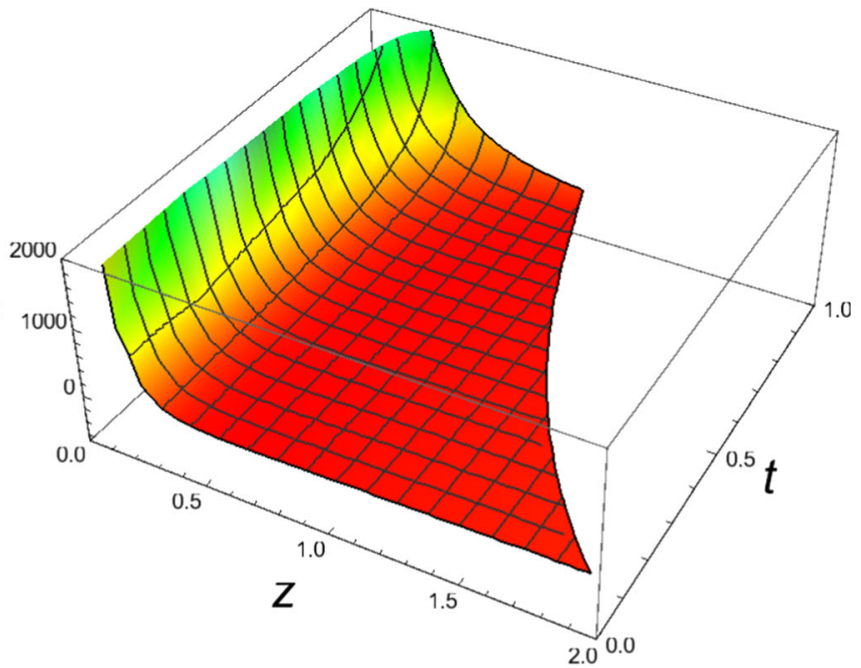

int 8 order $\epsilon$

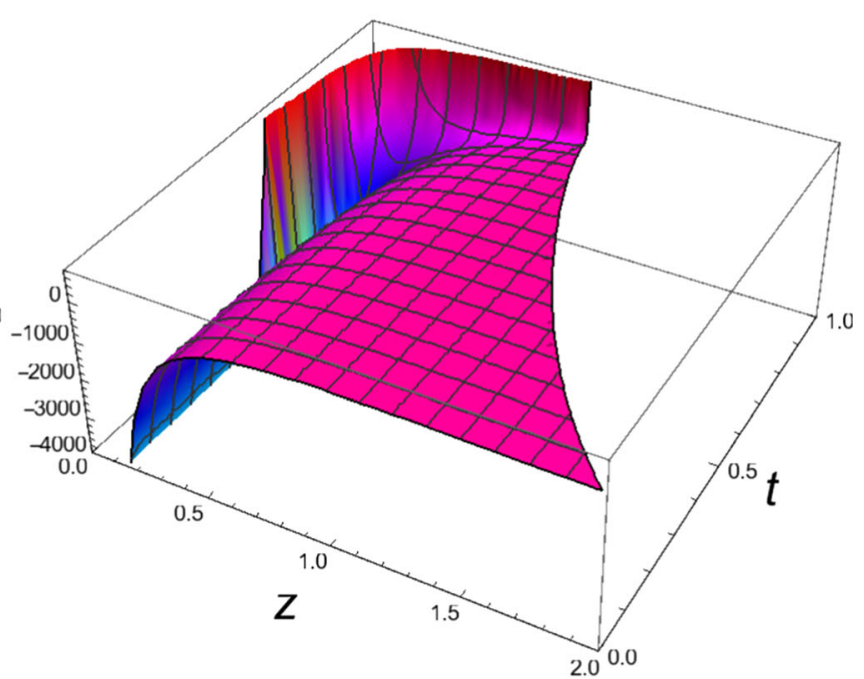

int 10 order $\epsilon^{2}$

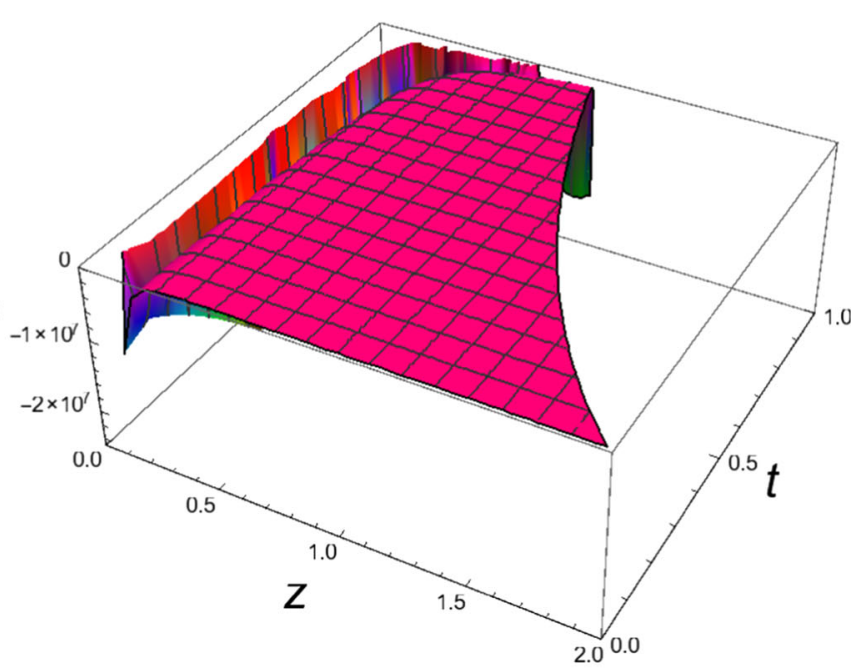

int 12 order $\epsilon$

Fig. 6 Plots of the first order in $\epsilon$ which contains GPLs of weight four for the MIs $\hat{I}_{7}, \ldots, \hat{I}_{12}$ 


\subsection{Integrals}

The simplest MI in the list is the two-loop phase space diagram shown in the top left panel of Fig. 2, for which one can find an expression which is exact in $d=4-2 \epsilon$ dimensions [51]

$$
\begin{aligned}
I_{1}= & 2^{-9+8 \epsilon} \pi^{-3+2 \epsilon} \frac{\Gamma^{3}(1-\epsilon)}{\Gamma(2-2 \epsilon) \Gamma(3-3 \epsilon)} \\
& \times m_{b}^{2}\left(\frac{m_{b}^{2}}{\mu^{2}}\right)^{-2 \epsilon}\left[z^{2}\left(1-t^{2}\right)\right]^{1-2 \epsilon}, \\
& \equiv m_{b}^{2} K(\epsilon)\left(\frac{m_{b}^{2}}{\mu^{2}}\right)^{-2 \epsilon} \hat{I}_{1}(z, t, \epsilon),
\end{aligned}
$$

with

$K(\epsilon)=2^{-9+8 \epsilon} \pi^{-3+2 \epsilon} \frac{\Gamma^{3}(1-\epsilon)}{\Gamma(2-2 \epsilon) \Gamma(3-3 \epsilon)}$.

The first two terms of the $\epsilon$ expansion of $\hat{I}_{1}$ read

$$
\begin{aligned}
\hat{I}_{1}= & z^{2}\left(1-t^{2}\right)-2 \epsilon z^{2}\left(1-t^{2}\right)[G(-1, t)+G(1, t) \\
& -2 G(0, y)+4 G(-1, y)]+\mathcal{O}\left(\epsilon^{2}\right) .
\end{aligned}
$$

In analogy with Eq. (3.6), we introduce the notation

$I_{i} \equiv m_{b}^{2} K(\epsilon)\left(\frac{m_{b}^{2}}{\mu^{2}}\right)^{-2 \epsilon} \hat{I}_{i}, \quad i \in\{2, \ldots, 12\}$.

The analytic expression of all $\hat{I}_{i}$ up to terms involving GPLs of weight four can be found in the ancillary file MasterIntegrals.txt.

We cross-checked 9 out of the 12 MIs which we calculated analytically in this work by comparing the numerical evaluation of their analytic expressions (carried out by means of $\mathrm{GiNaC}$ ) to the direct numerical integration of the MIs, carried out with the package SecDec [52-54]. We found agreement within the SecDec numerical integration error in all points tested. The remaining three MIs $\left(I_{8}, I_{11}, I_{12}\right)$ belong to subtopologies which also admit at least one two-particle cut. Consequently, these MIs cannot be evaluated directly by comparing them to the imaginary part of a $2 \rightarrow 2$ forward box calculated with SecDec, since that imaginary part receives a contribution from two-particle and three-particle cuts. Once the MIs corresponding to the two-particle cuts are known, they can be combined with the three-particle cuts calculated here and the sum of two- and three-particle cuts can finally be compared with the imaginary part of the corresponding $2 \rightarrow 2$ box integrals.

Finally, we employed GiNaC to evaluate the analytic results we found in all of the phase space. In Figs. 5 and
6 we plot, for all of the integrals $\hat{I}_{i}$, the order in $\epsilon$ at which GPLs of order four appear first.

\section{Conclusions}

In this paper we evaluated analytically the MIs needed for the calculation of the double emission corrections to the $b \rightarrow u W^{*}$ decay at tree level. The problem was mapped into the calculation of three-particle cuts in two-loop $b W^{*} \rightarrow$ $b W^{*}$ forward box diagrams. We identified a set of $12 \mathrm{MIs}$ belonging to three auxiliary topologies by using IBPs. The MIs depend on two dimensionless parameters. Their analytic expression in terms of GPLs was found by means of the DE method. The integrals can be evaluated with arbitrary numerical precision by means of the GPLs functions implemented in GiNaC. The complete analytic expression of all of the MIs can be found in an ancillary file included in the arXiv version of this paper. The result were cross checked against a direct numerical integration of the MIs carried out by means of the program SecDec. The results obtained here are needed for the analytic evaluation of the $b \rightarrow u W^{*}$ decay double differential distribution to NNLO in QCD. Since the two-loop virtual corrections to the $b \rightarrow u W^{*}$ decay are already known analytically $[14-16,18]$, the only missing element at this stage are the one-loop, single-emission diagrams contributing to $b \rightarrow u W^{*}$ decay to NNLO.

Acknowledgements The work of A.F. is supported in part by the National Science Foundation under Grant no. PHY-1417354 and PSC CUNY Research Award TRADA-48-265. A.B. and A.F. would like to thank G. Heinrich and S. Jahn for the help with the program SecDec. A.B. and A.F. would like to thank the Munich Institute for Astro- and Particle Physics (MIAPP) of the DFG cluster of excellence "Origin and Structure of the Universe" for hospitality and support.

Open Access This article is distributed under the terms of the Creative Commons Attribution 4.0 International License (http://creativecomm ons.org/licenses/by/4.0/), which permits unrestricted use, distribution, and reproduction in any medium, provided you give appropriate credit to the original author(s) and the source, provide a link to the Creative Commons license, and indicate if changes were made.

Funded by SCOAP $^{3}$.

\section{A MIs poles}

In this appendix we collect the explicit expression of the poles of the MIs which are divergent in the $\epsilon \rightarrow 0$ limit.

$$
\begin{aligned}
\hat{I}_{3}= & -\frac{1}{m_{b}^{4} \epsilon} \frac{8}{z t}[G(-1 ; t)-G(1 ; t)]+\mathcal{O}\left(\epsilon^{0}\right) . \\
\hat{I}_{6}= & \frac{1}{m_{b}^{6} \epsilon} \frac{32}{t z^{2}\left[4+z\left(t^{2}-1\right)\right]}[G(-1 ; t)-G(1 ; t)] \\
& +\mathcal{O}\left(\epsilon^{0}\right),
\end{aligned}
$$




$$
\begin{aligned}
\hat{I}_{7} & =\frac{1}{m_{b}^{8} \epsilon} \frac{128}{z^{4}\left(t^{2}-1\right)^{2}}+\mathcal{O}\left(\epsilon^{0}\right) . \\
\hat{I}_{8}= & -\frac{1}{m_{b}^{4} \epsilon} \frac{8}{z t}\left[G\left(-w_{2},-w_{1} ; t\right)+G\left(-w_{2}, w_{1} ; t\right)\right. \\
& -G\left(w_{2},-w_{1} ; t\right)-G\left(w_{2}, w_{1} ; t\right) \\
& -G\left(-1,-w_{1} ; t\right)-G\left(-1, w_{1} ; t\right)+G\left(1,-w_{1} ; t\right) \\
& +G\left(1, w_{1} ; t\right)+G\left(-w_{1},-1 ; t\right) \\
& -G\left(-w_{1}, 1 ; t\right)+G\left(w_{1},-1 ; t\right)-G\left(w_{1}, 1 ; t\right) \\
& +2 G(1 ; y) G\left(-w_{2} ; t\right)-2 G(1 ; y) G\left(w_{2} ; t\right) \\
& -G(0 ; y)\left(G(1 ; t)+G\left(-w_{2} ; t\right)-G\left(w_{2} ; t\right)\right) \\
& -G\left(-w_{2},-1 ; t\right)+G\left(w_{2}, 1 ; t\right) \\
& +\ln (2)\left(G\left(w_{2} ; t\right)-G\left(-w_{2} ; t\right)\right) \\
& +G(-1 ; t)(G(0 ; y)-2 G(1 ; y)+\ln (2)) \\
& +2 G(1 ; t) G(1 ; y)+G(-1,1 ; t)-G(1,-1 ; t) \\
& -\ln (2) G(1 ; t)]+\mathcal{O}\left(\epsilon^{0}\right) . \\
\hat{I}_{10} & =\frac{1}{m_{b}^{6} \epsilon} \frac{4}{t z}[G(-1 ; t)-G(1 ; t)]+\mathcal{O}\left(\epsilon^{0}\right) . \\
\hat{I}_{11}= & \frac{1}{m_{b}^{6} \epsilon^{2}} \frac{8}{t z^{2}}[G(-1 ; t)-G(1 ; t)] \\
& +\frac{1}{m_{b}^{6} \epsilon} \frac{4}{t z^{2}}\left\{2 \left[2 G\left(-1,-w_{1} ; t\right)+2 G\left(-1, w_{1} ; t\right)\right.\right. \\
& -2 G\left(1,-w_{1} ; t\right)-2 G\left(1, w_{1} ; t\right)+2 G\left(-w_{1},-1 ; t\right) \\
& -2 G\left(-w_{1}, 1 ; t\right) \\
& +2 G\left(w_{1},-1 ; t\right)-2 G\left(w_{1}, 1 ; t\right)-5 G(-1,-1 ; t) \\
& -G(-1,1 ; t)+2 G(0,-1 ; t) \\
& -2 G(0,1 ; t)+G(1,-1 ; t)+5 G(1,1 ; t)] \\
& -(G(-1 ; t)-G(1 ; t))[16 G(-1 ; y) \\
& -4 G(0 ; y)-8 G(1 ; y)+13+4 \ln (2)]\}+\mathcal{O}\left(\epsilon^{0}\right) . \\
& -2) \\
&
\end{aligned}
$$

$$
\begin{aligned}
\hat{I}_{12}= & \frac{1}{m_{b}^{8} \epsilon^{2}} \frac{128}{t\left(1-t^{2}\right) z^{4}}[G(-1 ; t)-G(1 ; t)] \\
& +\frac{1}{m_{b}^{8} \epsilon} \frac{64}{t\left(1-t^{2}\right) z^{4}}\{9 G(1,1 ; t)-9 G(-1,-1 ; t) \\
& +G(-1,1 ; t)+4 G(0,-1 ; t)-4 G(0,1 ; t) \\
& -G(1,-1 ; t)+(G(-1 ; t)-G(1 ; t)) \\
& \times[8 G(0 ; y)-16 G(-1 ; y)-13]\}+\mathcal{O}\left(\epsilon^{0}\right) .
\end{aligned}
$$

\section{References}

1. I.I.Y. Bigi, N.G. Uraltsev, A.I. Vainshtein, Nonperturbative corrections to inclusive beauty and charm decays: QCD versus phenomenological models. Phys. Lett. B 293, 430-436 (1992). arXiv:hep-ph/9207214
2. B. Blok, L. Koyrakh, M.A. Shifman, A.I. Vainshtein, Differential distributions in semileptonic decays of the heavy flavors in QCD. Phys. Rev. D 49, 3356 (1994). arXiv:hep-ph/9307247

3. A.V. Manohar, M.B. Wise, Inclusive semileptonic B and polarized Lambda(b) decays from QCD. Phys. Rev. D 49, 1310-1329 (1994). arXiv:hep-ph/9308246

4. M. Gremm, A. Kapustin, Order $1 / \mathrm{m}(\mathrm{b}) * * 3$ corrections to $B \rightarrow X_{c}$ lepton anti-neutrino decay and their implication for the measurement of Lambda-bar and lambda(1). Phys. Rev. D 55, 6924-6932 (1997). arXiv:hep-ph/9603448

5. M. Neubert, Analysis of the photon spectrum in inclusive $B \rightarrow X_{s} \gamma$ decays. Phys. Rev. D 49, $4623-4633$ (1994). arXiv:hep-ph/9312311

6. M. Neubert, QCD based interpretation of the lepton spectrum in inclusive $\bar{B} \rightarrow X_{u}$ lepton anti-neutrino decays. Phys. Rev. D 49, 3392-3398 (1994). arXiv:hep-ph/9311325

7. I.I.Y. Bigi, M.A. Shifman, N.G. Uraltsev, A.I. Vainshtein, On the motion of heavy quarks inside hadrons: Universal distributions and inclusive decays. Int. J. Mod. Phys. A 9, 2467-2504 (1994). arXiv:hep-ph/9312359

8. C.W. Bauer, Z. Ligeti, M.E. Luke, Precision determination of |V(ub)| from inclusive decays. Phys. Rev. D 64, 113004 (2001). arXiv:hep-ph/0107074

9. C.W. Bauer, Z. Ligeti, M.E. Luke, A Model independent determination of |V(ub)|. Phys. Lett. B 479, 395-401 (2000). arXiv:hep-ph/0002161

10. F. De Fazio, M. Neubert, B $\rightarrow \mathrm{X}(\mathrm{u})$ lepton anti-neutrino lepton decay distributions to order $\alpha_{s}$. JHEP 06, 017 (1999). arXiv:hep-ph/9905351

11. M. Brucherseifer, F. Caola, K. Melnikov, On the $O\left(\alpha_{s}^{2}\right)$ corrections to $b \rightarrow X_{u} e \bar{v}$ inclusive decays. Phys. Lett. B 721, 107-110 (2013). arXiv: 1302.0444

12. M. Brucherseifer, F. Caola, K. Melnikov, $\mathcal{O}\left(\alpha_{s}^{2}\right)$ corrections to fully-differential top quark decays. JHEP 04, 059 (2013). arXiv: 1301.7133

13. J. Gao, C.S. Li, H.X. Zhu, Top quark decay at next-to-nextto leading order in QCD. Phys. Rev. Lett. 110, 042001 (2013). arXiv: 1210.2808

14. G. Bell, NNLO corrections to inclusive semileptonic B decays in the shape-function region. Nucl. Phys. B 812, 264-289 (2009). arXiv:0810.5695

15. H.M. Asatrian, C. Greub, B.D. Pecjak, NNLO corrections to anti-B $\rightarrow \mathrm{X}(\mathrm{u}) 1$ anti-nu in the shape-function region. Phys. Rev. D 78, 114028 (2008). arXiv:0810.0987

16. M. Beneke, T. Huber, X.Q. Li, Two-loop QCD correction to differential semi-leptonic $\mathrm{b} \rightarrow \mathrm{u}$ decays in the shape-function region. Nucl. Phys. B 811, 77-97 (2009). arXiv:0810.1230

17. C. Greub, M. Neubert, B.D. Pecjak, NNLO corrections to $\bar{B} \rightarrow$ $X_{u} l \bar{v}_{l}$ and the determination of $|\mathrm{V}(\mathrm{ub})| \mathrm{l}$. Eur. Phys. J. C 65, 501-515 (2010). arXiv:0909.1609

18. R. Bonciani, A. Ferroglia, Two-loop QCD corrections to the heavyto-light quark decay. JHEP 11, 065 (2008). arXiv:0809.4687

19. C. Anastasiou, K. Melnikov, Higgs boson production at hadron colliders in NNLO QCD. Nucl. Phys. B 646, 220-256 (2002). arXiv:hep-ph/0207004

20. K. Melnikov, A. Mitov, Perturbative heavy quark fragmentation function through $\mathcal{O}\left(\alpha_{s}^{2}\right)$. Phys. Rev. D 70, 034027 (2004). arXiv:hep-ph/0404143

21. K. Melnikov, A. Mitov, The photon energy spectrum in $B \rightarrow X_{s}+$ $\gamma$ in perturbative QCD through $\mathcal{O}\left(\alpha_{s}^{2}\right)$. Phys. Lett. B 620, 69-79 (2005). arXiv:hep-ph/0505097

22. H.M. Asatrian, T. Ewerth, A. Ferroglia, P. Gambino, C. Greub, Magnetic dipole operator contributions to the photon energy spectrum in anti-B $\rightarrow \mathrm{X}(\mathrm{s})$ gamma at $\mathrm{O}($ alpha**2(s)). Nucl. Phys. B 762, 212-228 (2007). arXiv:hep-ph/0607316 
23. H.M. Asatrian, T. Ewerth, A. Ferroglia, C. Greub, G. Ossola, Complete $\left(O_{7}, O_{8}\right)$ contribution to $B \rightarrow X_{s} \gamma$ at order $\alpha_{s}^{2}$. Phys. Rev. D 82, 074006 (2010). arXiv: 1005.5587

24. C. Anastasiou, C. Duhr, F. Dulat, F. Herzog, B. Mistlberger, Higgs boson gluon-fusion production in QCD at three loops. Phys. Rev. Lett. 114, 212001 (2015). arXiv: 1503.06056

25. R. Bonciani, F. Buccioni, R. Mondini, A. Vicini, Double-real corrections at $\mathcal{O}\left(\alpha \alpha_{s}\right)$ to single gauge boson production. Eur. Phys. J. C 77, 187 (2017). arXiv:1611.00645

26. R.E. Cutkosky, Singularities and discontinuities of Feynman amplitudes. J. Math. Phys. 1, 429-433 (1960)

27. F. Tkachov, A theorem on analytical calculability of four loop renormalization group functions. Phys. Lett. B 100, 65-68 (1981)

28. K. Chetyrkin, F. Tkachov, Integration by parts: the algorithm to calculate beta functions in 4 loops. Nucl. Phys. B 192, 159-204 (1981)

29. S. Laporta, High precision calculation of multiloop Feynman integrals by difference equations. Int. J. Mod. Phys. A 15, 5087-5159 (2000). arXiv:hep-ph/0102033

30. C. Anastasiou, A. Lazopoulos, Automatic integral reduction for higher order perturbative calculations. JHEP 07, 046 (2004). arXiv:hep-ph/0404258

31. R.N. Lee, Presenting LiteRed: a tool for the Loop InTEgrals REDuction. arXiv: 1212.2685

32. R.N. Lee, LiteRed 1.4: a powerful tool for reduction of multiloop integrals. J. Phys. Conf. Ser. 523, 012059 (2014). arXiv:1310.1145

33. P. Maierhoefer, J. Usovitsch, P. Uwer, Kira-A Feynman Integral Reduction Program. arXiv:1705.05610

34. A.V. Smirnov, Algorithm FIRE-Feynman Integral REduction. JHEP 10, 107 (2008). arXiv:0807.3243

35. A.V. Smirnov, V.A. Smirnov, FIRE4, LiteRed and accompanying tools to solve integration by parts relations. Comput. Phys. Commun. 184, 2820-2827 (2013). arXiv:1302.5885

36. A.V. Smirnov, FIRE5: a C++ implementation of Feynman Integral REduction. Comput. Phys. Commun. 189, 182-191 (2014). arXiv: 1408.2372

37. C. Studerus, Reduze-Feynman integral reduction in C++. Comput. Phys. Commun. 181, 1293-1300 (2010). arXiv:0912.2546

38. A. von Manteuffel, C. Studerus, Reduze 2-Distributed Feynman Integral Reduction. arXiv:1201.4330

39. A. Kotikov, Differential equations method: new technique for massive Feynman diagrams calculation. Phys. Lett. B 254, 158-164 (1991)
40. E. Remiddi, Differential equations for Feynman graph amplitudes. Nuovo Cim. A 110, 1435-1452 (1997). arXiv:hep-th/9711188

41. T. Gehrmann, E. Remiddi, Differential equations for two loop four point functions. Nucl. Phys. B 580, 485-518 (2000). arXiv:hep-ph/9912329

42. M. Argeri, P. Mastrolia, Feynman diagrams and differential equations. Int. J. Mod. Phys. A 22, 4375-4436 (2007). arXiv:0707.4037

43. J.M. Henn, Lectures on differential equations for Feynman integrals. J. Phys. A 48, 153001 (2015). arXiv:1412.2296

44. A. Goncharov, Polylogarithms in arithmetic and geometry. Proc. Int. Congr. Math. 1(2), 374-387 (1995)

45. A. B. Goncharov, Multiple polylogarithms and mixed Tate motives. arXiv:math/0103059

46. E. Remiddi, J.A.M. Vermaseren, Harmonic polylogarithms. Int. J. Mod. Phys. A 15, 725-754 (2000). arXiv:hep-ph/9905237

47. J.M. Henn, Multiloop integrals in dimensional regularization made simple. Phys. Rev. Lett. 110, 251601 (2013). arXiv:1304.1806

48. J. Vollinga, S. Weinzierl, Numerical evaluation of multiple polylogarithms. Comput. Phys. Commun. 167, 177 (2005). arXiv:hep-ph/0410259

49. A.B. Goncharov, Multiple polylogarithms, cyclotomy and modular complexes. Math. Res. Lett. 5, 497-516 (1998). arXiv:1105.2076

50. T. Gehrmann, E. Remiddi, Two loop master integrals for $\gamma * \rightarrow$ 3 jets: the planar topologies. Nucl. Phys. B 601, 248-286 (2001). arXiv:hep-ph/0008287

51. A. Gehrmann-De Ridder, T. Gehrmann, G. Heinrich, Four particle phase space integrals in massless QCD. Nucl. Phys. B 682, 265288 (2004). arXiv:hep-ph/0311276

52. J. Carter, G. Heinrich, SecDec: a general program for sector decomposition. Comput. Phys. Commun. 182, 1566-1581 (2011). arXiv: 1011.5493

53. S. Borowka, G. Heinrich, S.P. Jones, M. Kerner, J. Schlenk, T. Zirke, SecDec-3.0: numerical evaluation of multi-scale integrals beyond one loop. Comput. Phys. Commun. 196, 470-491 (2015). arXiv:1502.06595

54. S. Borowka, G. Heinrich, S. Jahn, S.P. Jones, M. Kerner, J. Schlenk et al., pySecDec: a toolbox for the numerical evaluation of multiscale integrals. Comput. Phys. Commun. 222, 313-326 (2018). arXiv:1703.09692 